\title{
Are fibre posts associated with the occurrence of prosthetic complications?
}

\author{
Abstracted from \\ Sorrentino R, Di Mauro MI, Ferrari M, Leone R, Zarone F. \\ Complications of endodontically treated teeth restored with fiber posts and single crowns or fixed dental prostheses-a systematic review. Clin Oral \\ Investig 2016; 20: 1449-1457. doi: 10.1007/s00784-016-1919-8. Epub 2016 Jul 26. Review. PubMed PMID: 27460566. \\ Address for correspondence: Roberto Sorrentino, Department of Medical Biotechnologies, Division of Fixed Prosthodontics, University of Siena, \\ Siena, Italy. E-mail errestino@libero.it
}

\section{Question: Is there a relationship between the presence of fibre posts in abutment teeth and the occurrence of prosthetic complications in single crowns (SCs) or fixed prostheses (FDPs)?}

Data sources The Pubmed, Evidence-Based Dentistry, BMJ Clinical Evidence, EmbaseDynamed, and www.opengrey.eu databases and manual search of reference lists.

Study selection Randomised clinical trials (RCTs) were accepted if they had: participants with no periodontal disease and teeth restored with SCs or FDPs and compared fibre posts and other prosthetic systems and evaluated the prosthetic complications with a minimum observational period of 36 months.

Data extraction and synthesis Titles and abstracts were evaluated independently by two reviewers, any disagreement was discussed with a third reviewer. The agreement for the two reviewers was $97 \%$. The quality and the risk of bias of the studies included was assessed following the Cochrane Handbook considering the domains of randomisation, sample size, inclusion and exclusion criteria, followup achieved, blinding, withdrawing and groups' compatibility for quality assessment, and for the risk of bias the domains evaluated were allocation concealment, blinding of outcome assessor and follow-up. Results The database search yielded 4,230 records; after duplications removal, 3,670 records were reviewed independently by the authors, and four articles were chosen to include in the systematic review. The most frequently reported failures in the available studies were as follows: fibre post debonding, loss of retention of single crowns and marginal gaps. Less frequently, chippings and fractures were recorded in SCs. No studies about complications related to FDPs were found. The failure rate ranged from 0 to $28.2 \%$.

Conclusions A correlation between the failure rates of fibre posts and the type of prosthetic restoration (SCs or FDPs) cannot be found. Further RCTs are required to achieve evidence-based conclusions, particularly about the use of fibre posts with FDPs.

\section{Commentary}

Endodontic treatment is a viable therapy to restore a tooth in presence of extensive dental caries or pulp infection. Endodontically treated teeth usually present lower amount of remaining dental structure, so to get prosthetic stability and retention, after the removal of necrotic dental tissue, intraradicular posts are one possible option. However, intraradicular posts can lead to some complications such as root fracture, crown/ restoration fracture, debonding and fracture of the post, crown dislodgement, post-core-crown complex loosening. . $2,3^{2,3}$

The systematic review had the aim of investigating the relationships between the presence of fibre posts in abutment teeth and the occurrence of endodontic and prosthetic complications in the presence of single crowns and fixed dental prostheses, in patients with periodontal status ad integrum.

The review included studies that fulfill the PICO question, however, some of the characteristics of the included studies are not similar. The selection of tooth type varies - two studies included only premolars and the other two studies included any tooth, but no study specified if the tooth is mandibular or maxillary. Furthermore, the amount of residual coronal structure analysed is discrepant among the four studies and they do not use the same parameters (walls/ferrule/\%/Black classification).

The quality and heterogeneity of the four RCTs may be questionable. The four studies did not calculate the sample size, the authors categorised other studies at high risk of bias due to problems with blinding and withdrawing. The studies also have different follow-ups ranging from 36 to 72 months and moreover the operator in each study has a different type of experience (undergraduate students/endodontist).

The authors have to present the results in a narrative assessment since the results extracted and the variability among the studies preclude them to perform a meta-analysis.

For what the authors conclude fibre post debonding and crown dislodgment is the most frequently reported outcome and the preservation of tooth structure seems to reduce the risk of failure, however, the results with some caveats should be interpreted with caution due to the limited quality of the available evidence presented by the review.

\section{Natacha Reis, Marco Bergamini, Thamar Silvestre and Analia Veitz-Keenan} New York University, College of Dentistry, New York, USA

1. Guldener KA, Lanzrein CL, Guldener BE, Lang NP, Ramseier CA, Salvi GE. Long-term Clinical Outcomes of Endodontically Treated Teeth Restored with or without Fiber Post-retained Single-unit Restorations. J Endod 2017; 43: 188-193.

2. Sarkis-Onofre R, Fergusson D, Cenci MS, Moher D, Pereira-Cenci T. Performance of Post-retained Single Crowns: A Systematic Review of Related Risk Factors. J Endod 2017; 43: 175-183.

3. Marchionatti AME, Wandscher VF, Rippe MP, Kaizer OB, Valandro LF. Clinical performance and failure modes of pulpless teeth restored with posts: a systematic review. Braz Oral Res 2017; 31: http://dx.doi.org/10.1590/1807-3107bor-2017. vol31.0064.

Evidence-Based Dentistry (2018) 19, 62. doi:10.1038/sj.ebd.6401313 